\title{
Magnesium-Magnetic Field Synergy Enhances Mouse Bone Marrow Mesenchymal Stem Cell Differentiation into Osteoblasts Via the MAGT1 Channel
}

\author{
Yifan Wang $\left(\mathbb{D},{ }^{1}\right.$ Xin Wu $\left(\mathbb{D},{ }^{1}\right.$ Wenjing Yang $\left(\mathbb{D},{ }^{2}\right.$ Pei Feng $\left(\mathbb{D},{ }^{3}\right.$ Wei Tan $\left(\mathbb{D},{ }^{1}\right.$ Youwen Deng $\left(\mathbb{D},,^{1}\right.$ \\ and Cijun Shuai $\mathbb{D}^{2,3}$ \\ ${ }^{1}$ Department of Spine Surgery, Third Xiangya Hospital, Central South University, Changsha, 410013 Hunan, China \\ ${ }^{2}$ Institute of additive Manufacturing, Jiangxi University of Science and Technology, Nanchang 330013, China \\ ${ }^{3}$ State Key Laboratory of High-Performance Complex Manufacturing, College of Mechanical and Electrical Engineering, \\ Central South University, Changsha 410083, China
}

Correspondence should be addressed to Youwen Deng; drywdeng@163.com and Cijun Shuai; shuai@csu.edu.cn

Received 28 December 2021; Revised 12 February 2022; Accepted 14 February 2022; Published 3 March 2022

Academic Editor: Xiaoming Li

Copyright (c) 2022 Yifan Wang et al. This is an open access article distributed under the Creative Commons Attribution License, which permits unrestricted use, distribution, and reproduction in any medium, provided the original work is properly cited.

\begin{abstract}
Magnesium ion $\left(\mathrm{Mg}^{2+}\right)$-based materials are known to exert osteogenic effects that can be enhanced by the bioelectrical properties of magnetic fields. In this study, we examined the effect of a medium-strength static magnetic field (SMF), combined with a $\mathrm{Mg}^{2+}$ containing medium, on the proliferation and osteogenic differentiation of mouse bone marrow mesenchymal stem cells (BMSCs). Mouse BMSCs were divided into a control group, $7.5 \mathrm{mM} \mathrm{Mg}^{2+}$ group, $15 \mathrm{mT}$ SMF group, and $7.5 \mathrm{mM} \mathrm{Mg}^{2+}$ plus $15 \mathrm{mT}$ SMF group. Osteoblast proliferation was measured using a Cell Counting Kit-8 assay, whereas osteogenic differentiation was detected using alkaline phosphatase (ALP) staining and western blot analysis, respectively. The number and size of calcium nodules were determined using Alizarin Red staining. Compared with those in the control group, the ALP activity, calcium nodule formation, and osteogenic protein expression were promoted in other groups. In particular, $\mathrm{Mg}^{2+}-\mathrm{SMF}_{\mathrm{Sad}} \mathrm{a}$ significant effect after 7 days of intervention and more effectively promoted BMSC differentiation and proliferation than either $\mathrm{Mg}^{2+}$ or the SMF alone, suggesting that $\mathrm{Mg}^{2+}-\mathrm{SMF}$ synergistically contributed to osteogenic differentiation and cell proliferation. To examine their roles in bone differentiation, the Magt1 and Creb1 genes were silenced in BMSCs, and the findings indicated that the synergistic intervention with $\mathrm{Mg}^{2+}$ and magnetic fields might exert osteogenic effects via the MAGT1 channel and CREB1 protein. This study provides an experimental basis for a potential $\mathrm{Mg}^{2+}-$ SMF synergistic artificial bone material that could be clinically applied in the treatment of bone defects.
\end{abstract}

\section{Introduction}

Magnesium ion $\left(\mathrm{Mg}^{2+}\right)$-based materials are expected to be applied in clinical practice, as they exert osteogenic effects and their mechanical properties are similar to those of the natural bone. Unfortunately, the rate of degradation of pure $\mathrm{Mg}$ based materials that are used in orthopedics is extremely high. In the bone tissue, wherein fluid circulation and metabolism are relatively slow, this degradation can cause excessive local $\mathrm{Mg}^{2+}$ and hydrogen accumulation, resulting in a highly alkaline environment, which adversely affects the blood supply to surrounding soft tissues as well as cell adhesion and the repair of bone defects.

Biodegradable orthopedic materials have been extensively studied in recent years [1,2], and numerous studies have shown that $\mathrm{Mg}^{2+}$ can promote the proliferation and osteogenic differentiation of bone marrow mesenchymal stem cells (BMSCs) [3-6]. In addition to promoting the differentiation of BMSCs into osteoblasts, $\mathrm{Mg}^{2+}$ can increase extracellular matrix (ECM) mineralization, resulting in excellent osteoinduction [7-9]. A previous study has confirmed that BMSCs display the best proliferation and 
osteogenic differentiation when cultured with $7.5 \mathrm{mM} \mathrm{Mg}^{2+}$ [10]. In particular, $\mathrm{Mg}^{2+}$ can promote osteogenic differentiation by inducing CAMP responsive element binding protein 1 (CREB1) phosphorylation via the $\mathrm{Mg}^{2+}$ channel magnesium transporter 1 (MAGT1) [9]. $\mathrm{Mg}^{2+}$ can also induce BMSC differentiation into osteoblasts directly, via MAGT1, and promote the expression of intracellular osteogenic signaling molecules [alkaline phosphatase (ALP), OCN, COL1, and RUNX2]. Since osteogenic properties of $\mathrm{Mg}^{2+}$ are associated with its transmembrane transport, it is important to determine the mechanisms underlying the opening of the MAGT1 channel to induce $\mathrm{Mg}^{2+}$ influx. Therefore, an understanding of how to effectively open the MAGT1 channel in the cell membrane and cause an effective influx of $\mathrm{Mg}^{2+}$ is the key to achieving the full osteoinductive activity of $\mathrm{Mg}^{2+}$.

The magnetic field environment can change the opening frequency of $\mathrm{Mg}^{2+}$ channel proteins on the cell membrane surface. Thus, the influx efficiency of $\mathrm{Mg}^{2+}$ can be improved to enhance its biological effect on osteogenesis. Numerous studies have shown that BMSC proliferation and osteoinduction can be accelerated by medium-strength magnetic fields (1 mT-1 T) [11-14], which include both pulsed electromagnetic fields (PEMFs) [15-17] and static magnetic fields (SMFs) [18-22]. Since magnetic fields are noninvasive and safe, they have the potential for broad clinical applications [23-27]. Although PEMFs are currently extensively studied for the induction of bone formation, SMFs have unique advantages for the preparation of artificial bone materials. For example, SMFs do not require powerful equipment for the biophysical stimulation of BMSCs, and it is easier to produce osteogenic effects when magnetic materials are added to artificial bone materials. SMFs can also exert important regulatory effects during bone metabolism and remodeling and have become an important biophysical tool for treating nonunions and promoting bone healing [28-30]. Studies have shown that SMFs can promote bone repair, bone deposition, and bone formation in vitro and in vivo [31-34]. In addition, SMFs can inhibit the reduction of the bone density caused by surgery or prostheses. Magnetic fields mainly exert osteogenic effects on BMSCs via electrical and mechanical receptors on the cell membrane that convert bioelectrical signals into biochemical signals, thereby activating intracellular signaling cascades. Indeed, osteogenesis-related ion channels, such as MAGT1, TWIKrelated $\mathrm{K}^{+}$channel 1 , and ORAI calcium release-activated calcium modulator $1 / 2$, have been shown to alter their opening frequencies under the action of magnetic fields, thereby promoting osteogenesis [35-37]. However, it remains unclear whether the bioelectrical effects of a magnetic field could be combined with $\mathrm{Mg}^{2+}$ to regulate the osteogenic properties of ion channels and thereby synergistically enhance osteogenic differentiation and ECM mineralization.

In this study, we aimed to combine two factors with recognized osteogenic effects, $\mathrm{Mg}^{2+}$ and SMFs, in order to explore whether they can synergistically enhance bone formation while eliminating the disadvantages of $\mathrm{Mg}^{2+}$ as a potential new biomaterial. The findings of this study provide an experimental basis for a putative new type of $\mathrm{Mg}^{2+}-\mathrm{SMF}$ synergistic artificial bone material with excellent osteogenic properties.

\section{Materials and Methods}

2.1. Cell Culture. BMSCs were purchased from Cyagen, Inc. (Beijing, China) and were grown in a BMSC growth medium at $37^{\circ} \mathrm{C}$ with $5 \% \mathrm{CO}_{2}$. The cells were cultured in Dulbecco's modified Eagle's medium (DMEM) containing 10\% fetal bovine serum (FBS) and 1\% penicillin and streptomycin in T25 culture flasks for expansion. To induce osteogenic differentiation, BMSCs were cultured in DMEM with $50 \mathrm{mM}$ ascorbic acid, $10 \mathrm{mM}$ dexamethasone, and $10 \mathrm{mM} \beta$-glycerophosphate (Sigma-Aldrich, St. Louis, MO, USA) at $37^{\circ} \mathrm{C}$ with $5 \% \mathrm{CO}_{2}$. The cells were divided into the following four groups: control $\left(0 \mathrm{mMMg}^{2+}\right.$ and $\left.0 \mathrm{mT} \mathrm{SMF}\right), \mathrm{Mg}^{2+}$ $\left(7.5 \mathrm{mM} \mathrm{Mg}^{2+}\right)$, SMF (15 $\left.\mathrm{mT} \mathrm{SMF}\right)$, and $\mathrm{Mg}^{2+}-\mathrm{SMF}$ (7.5 $\mathrm{mM} \mathrm{Mg}^{2+}$ combined with $15 \mathrm{mT} \mathrm{SMF)} \mathrm{(Figure} \mathrm{1(a)),}$ and their proliferation and osteogenic differentiation were observed. $\mathrm{Mg}^{2+}$ environment was added to the osteogenic induction medium in an appropriate proportion by anhydrous magnesium sulfate powder (aladdin囚Shanghai, China), and sterilized by filtration with a $0.22 \mu \mathrm{m} / 28 \mathrm{~mm}$ filter (Beyotime, Shanghai, China).

2.2. Gene Silencing. Two genes (Magt1 and Creb1) were selected for silencing to verify their roles in the synergistic osteogenic effect of $\mathrm{Mg}^{2+}$ and the SMF. The same gene silencing method was used for both genes. BMSCs were cultured in 24-well plates for $36 \mathrm{~h}$ to reach $30-50 \%$ confluence. Transfection was performed with target gene-specific small interfering RNAs (siRNAs) using a siRNA transfection kit (RIBOBIO, Guangdong, China). The grouping included a nonspecific control (nc), siRNA (si), $\mathrm{Mg}^{2+}-\mathrm{SMF}$ plus nonspecific control $\left(\mathrm{ms}+\mathrm{nc}\right.$ ), and $\mathrm{Mg}^{2+}-\mathrm{SMF}$ plus siRNA (ms $+\mathrm{si}$ ), as shown in Figure 1(b). After transfection, the cell plate was incubated at $37^{\circ} \mathrm{C}$ with $5 \% \mathrm{CO} 2$ for $48 \mathrm{~h}$. The efficiency of siRNA transfection was observed under a fluorescence microscope (Leica, Wetzlar, Germany); a 30-50\% proportion of fluorescently labeled cells indicated good transfection and successful silencing of the target gene. The cells were then cultured in different intervention environments for different times, and the expression levels of the osteogenic genes were evaluated in each group.

2.3. SMF Exposure. An SMF exposure system was produced using a neodymium $(\mathrm{Nd} 2 \mathrm{Fe} 14 \mathrm{~B})$ disc magnet $(2 \mathrm{~mm}$ thick, $35 \mathrm{~mm}$ in diameter; Xinhongchang Magnets, Guangdong, China) and a 6-well culture plate (Figure 2). Briefly, the magnetic disc was placed above a well to expose the culture to a north magnetic field, and its strength was altered by controlling the distance between the magnetic disc and the culture plate. To stimulate BMSC osteogenesis, we used the optimal magnetic field strength of $15 \mathrm{mT}$, as has been shown in a previous study [13]. A Gauss meter (TS200; Sanliang, Tokyo, Japan) was used to measure the SMF strength.

2.4. Cell Proliferation Assay. To measure cell proliferation, we used a Cell Counting Kit-8 (CCK-8; Dojindo, Kumamoto, Japan). Briefly, BMSCs were seeded into 96-well plates 


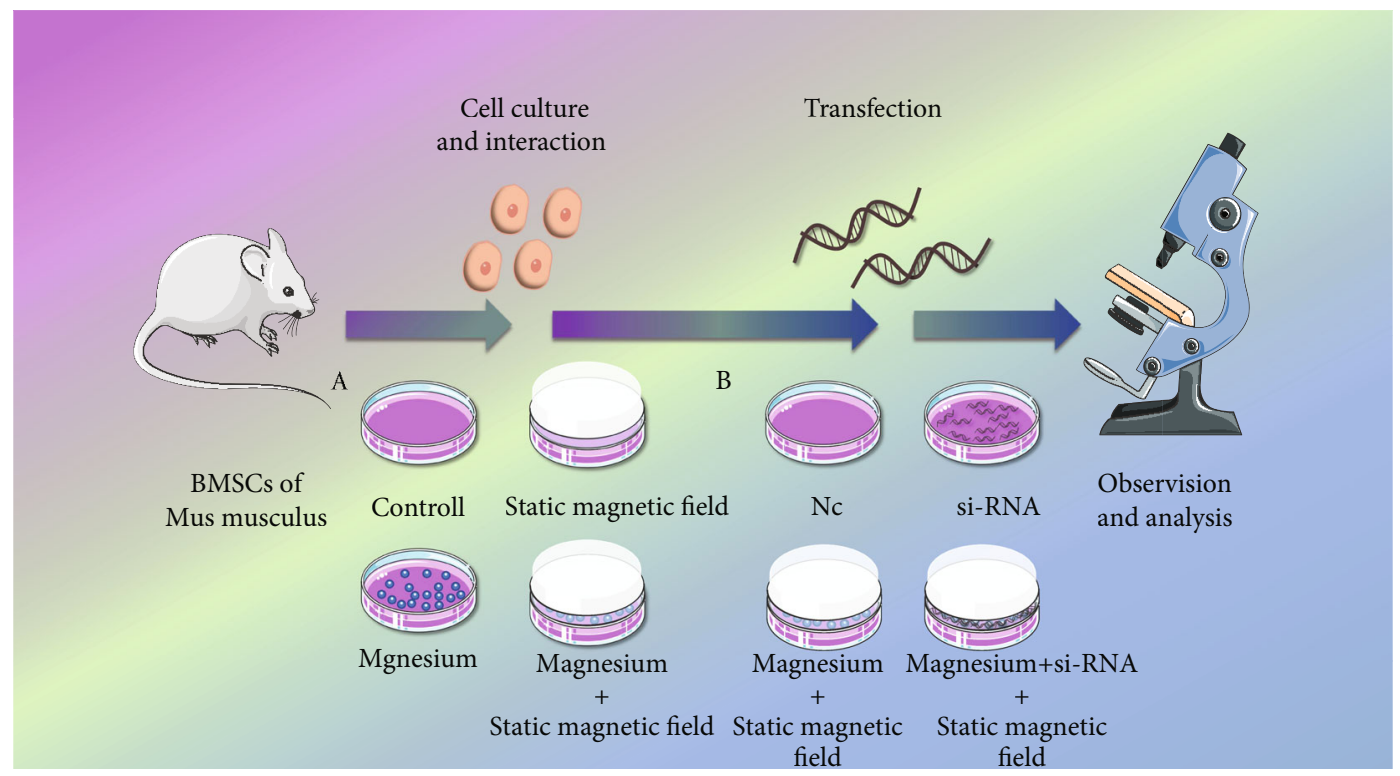

FIgURE 1: Study design. (a) BMSCs were divided into four groups: control, $\mathrm{Mg}^{2+}$, SMF, and $\mathrm{Mg}^{2+}-\mathrm{SMF}$. This grouping was done to compare the differences between the magnetic field intervention alone, the magnesium ion intervention and the synergistic intervention. (b) SiRNA transfection was used to silence genes in the following groups: non-specific control (nc), siRNA (si), $\mathrm{Mg}^{2+}$-SMF non-specific control (ms $+\mathrm{nc}$ ), and $\mathrm{Mg}^{2+}-\mathrm{SMF}$ siRNA $(\mathrm{ms}+\mathrm{si})$. $\mathrm{nc}$ and si to verify whether siRNA reagents are toxic to cells, $\mathrm{ms}+\mathrm{nc}$ and $\mathrm{ms}+\mathrm{si}$ to verify the role of target genes in synergistic osteogenesis.
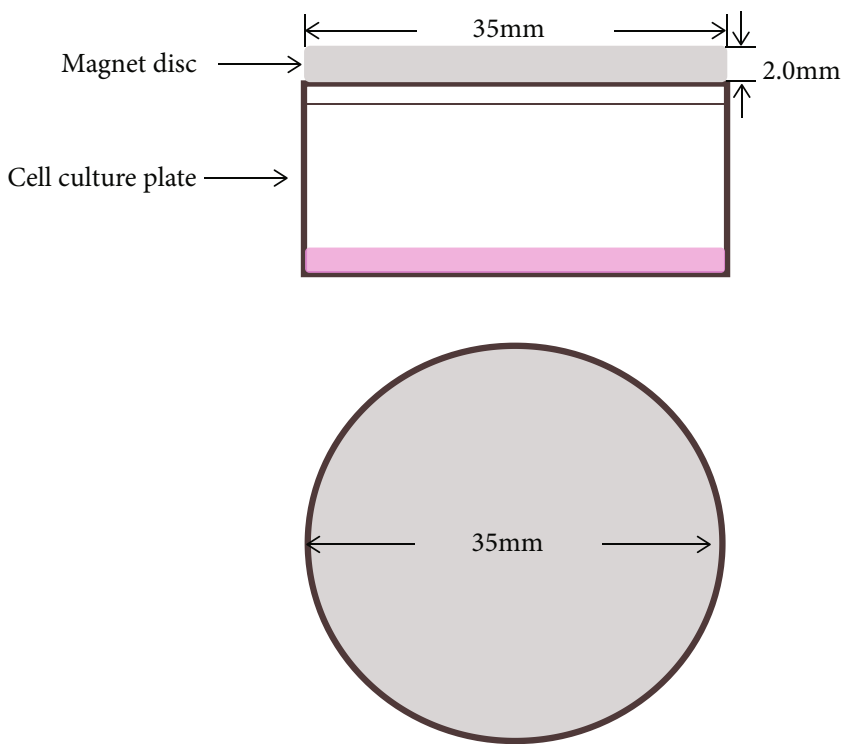

FIGURE 2: Schematic overview of SMF exposure system. A magnetic disc was placed above the wells of a 6-well culture plate to expose the culture to SMF.

$\left(2 \times 10^{3}\right.$ cells/well $)$ and cultured at $37^{\circ} \mathrm{C}$ with $5 \% \mathrm{CO}_{2}$ for 1 , 3,5 , or 7 days, with or without SMF exposure, in a medium with or without $7.5 \mathrm{mM} \mathrm{Mg}^{2+}$. Cells that were cultured without SMF or $\mathrm{Mg}^{2+}$ exposure were used as a negative control. After incubation of plates with the CCK- 8 reagents for $2 \mathrm{~h}$, the absorbance was measured at $450 \mathrm{~nm}$ using a microplate reader (Bio-Rad, Hercules, CA, USA).

2.5. ALP Staining. BMSCs were seeded into a 6-well plate and cultured for 2 days to reach confluence. The cells were then cultured in an osteogenic medium containing DMEM supplemented with 10\% FBS (Invitrogen. Waltham, MA, USA) and $50 \mu \mathrm{g} / \mathrm{mLL}$-ascorbic acid (Sigma-Aldrich) at $37^{\circ} \mathrm{C}$ with $5 \% \mathrm{CO}_{2}$ for 7 days, with or without the SMF and/or $\mathrm{Mg}^{2+}$, and the osteogenic medium was changed every 2 days. ALP staining was performed using a 5-bromo-4chloro-3-indolylphosphate/nitro blue tetrazolium ALP color development kit (Beyotime, Shanghai, China) according to the manufacturer's instructions, and the cells were then observed under an optical microscope.

2.6. Alizarin Red Staining. Osteoblast differentiation was measured by quantifying the formation of mineralized bone nodules using an Alizarin Red staining assay. Briefly, BMSCs were seeded into a 6 -well plate $\left(4 \times 10^{4}\right.$ cells/well $)$ and cultured for 2 days to reach confluence. The cells were then cultured in the osteogenic medium at $37 \pm 8^{\circ} \mathrm{C}$ with $5 \% \mathrm{CO}_{2}$ for 30 days, with or without the SMF and/or $\mathrm{Mg}^{2+}$, then washed with phosphate-buffered saline, and stained with a $40 \mathrm{mM}$ Alizarin Red solution for $10 \mathrm{~min}$. After the cells were washed five times and decolorized with $10 \mathrm{mM}$ sodium phosphate containing $10 \%$ cetylpyridinium chloride for $15 \mathrm{~min}$ at $26^{\circ} \mathrm{C}$, Alizarin Red staining was quantified by Image $\mathrm{J}$ (National Institutes of Health, Bethesda, MD, USA).

2.7. Western Blot Analysis. ALP, RUNX2, OSX, and COL1 protein expression was measured using western blotting. Total protein was extracted from cells using radioimmunoprecipitation assay lysis buffer supplemented with $1 \%$ phenylmethanesulfonyl fluoride. Protein concentrations were measured using a Pierce bicinchoninic acid protein assay kit (Thermo Fisher Scientific, Waltham, MA, USA). Equal amounts of protein were separated by sodium dodecyl 

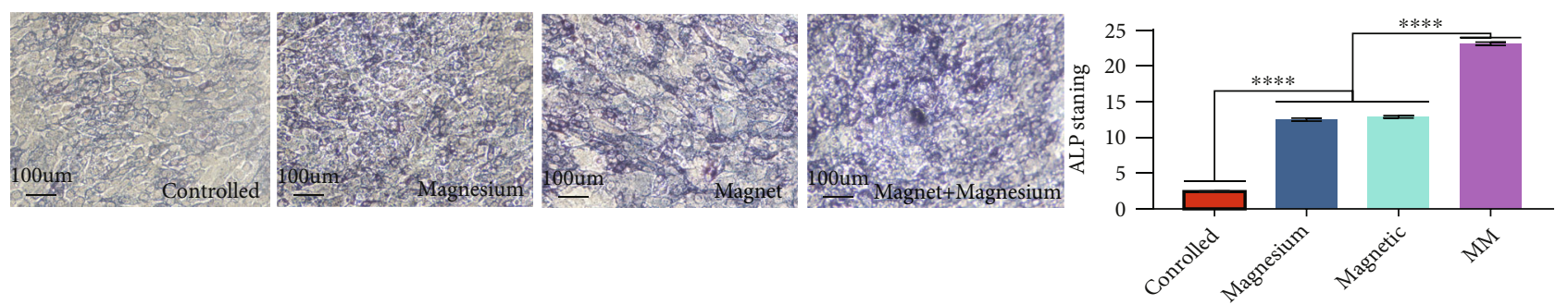

(a)
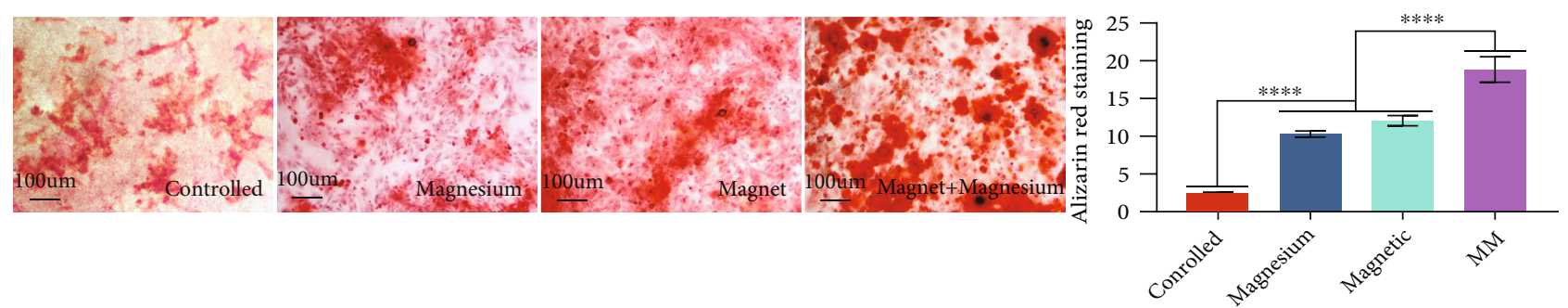

(b)

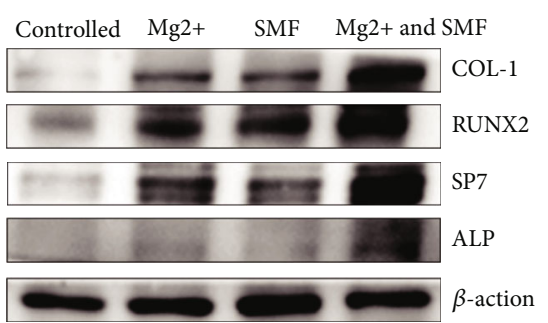

(c)

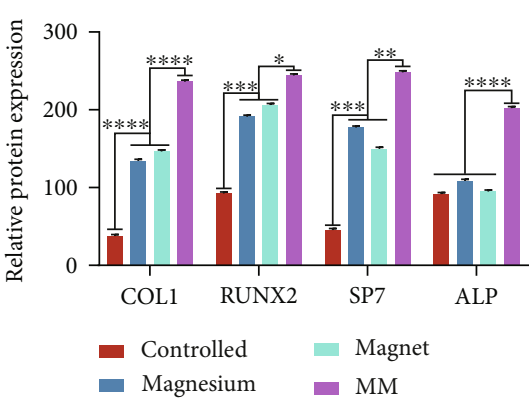

(d)

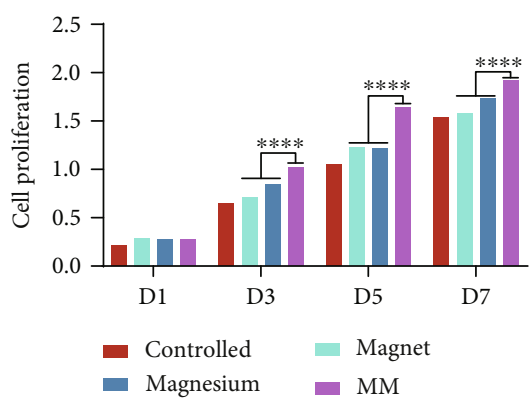

(e)

Figure 3: Osteogenic effects of $\mathrm{Mg}^{2+}$ and SMF. (a) Alp staining in BMSCs cultured for 7 days. $\mathrm{Mg}^{2+}$-SMF synergy promoted BMSCs ALP activity. (b) Alizarin red staining to detect matrix mineralization in BMSCs cultured for 28 days. The most calcium nodules were observed in BMSCs treated with $\mathrm{Mg}^{2+}$-SMF synergy. (c) Western blot analysis of protein expression in BMSCs cultured for 7 days. Mg ${ }^{2+}$ and SMF synergistically promoted the expression of BMSCs COL1, Runx2, SP7, and ALP. (d) Cell proliferation was assessed using CCK assays in BMSCs cultured for $1,3,5$, and 7 days. $\mathrm{Mg}^{2+}$-SMF synergy promoted BMSCs proliferation.

sulfate-polyacrylamide gel electrophoresis (10\%) and transferred onto polyvinylidene fluoride membranes, which were then blocked with skim milk for $1 \mathrm{~h}$ and incubated with the following antibodies (Abcam, Shanghai, China) overnight at $4^{\circ} \mathrm{C}$ : anti-ALP (ab83259), anti-RUNX2 (ab23981), anti-Sp7/ OSX (ab209484), and anti-collagen I (ab34710). The membranes were then washed three times with Tris-buffered saline containing 1\%o Tween 20 and incubated with IgG (heavy + light chains; ab205718) for $1 \mathrm{~h}$. Protein bands were visualized using enhanced chemiluminescence detection (the protein bands were photographed after incubation with a chromogenic solution). Relative protein levels were determined by normalizing their expression to that of $\beta$-actin (ab119716).

2.8. Statistical Analysis. All experiments were conducted at least three times. Data were processed using the GraphPad Prism 9 software (GraphPad Software, San Diego, CA, USA) and expressed as the mean \pm standard deviation. Differences between two groups were determined using the Stu- dent's $t$-test, and one-way analysis of variance with Tukey's multiple comparison test was used for multiple-group comparison. $P$-values of $<0.05$ were considered statistically significant.

\section{Results}

3.1. Effects of $\mathrm{Mg}^{2+}$ and the SMF on Differentiation and Proliferation of BMSCs. ALP staining showed (Figure 3(a)) that osteogenic differentiation was significantly enhanced in BMSCs treated with both $\mathrm{Mg}^{2+}$ and the SMF compared with that in BMSCs treated with either $\mathrm{Mg}^{2+}$ or the SMF alone and in the control group. Thus, the combination of $\mathrm{Mg}^{2+}$ and the SMF showed a synergistic effect and strongly promoted cell osteogenesis.

To determine the effect of the combination of $\mathrm{Mg}^{2+}$ with the SMF on ECM mineralization, BMSCs were stained with Alizarin Red after 28 days in culture. Cells treated with $\mathrm{Mg}^{2+}$ and the SMF in combination had the highest number of calcium nodules, while there was little difference in the number 


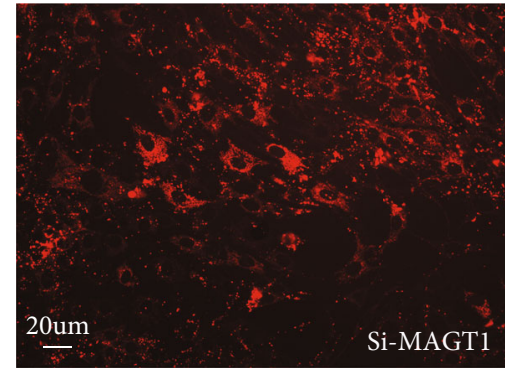

(a)
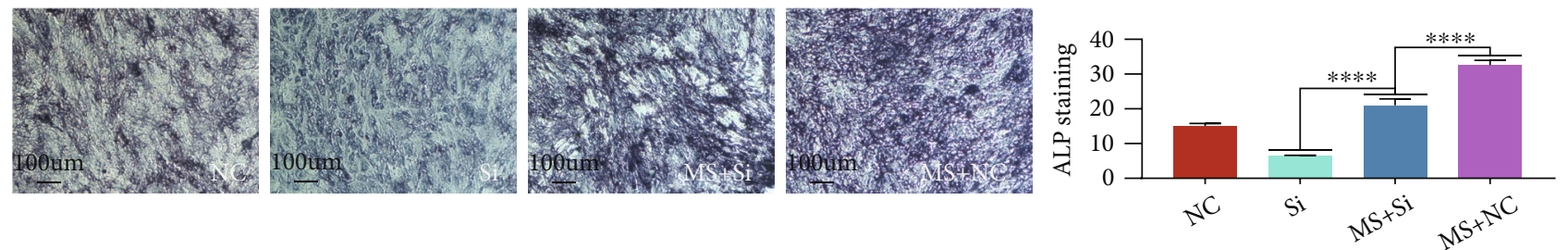

(b)
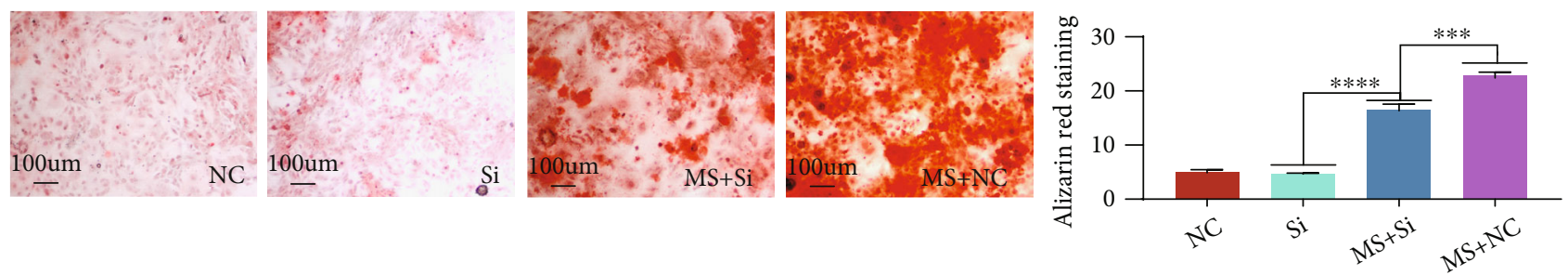

(c)

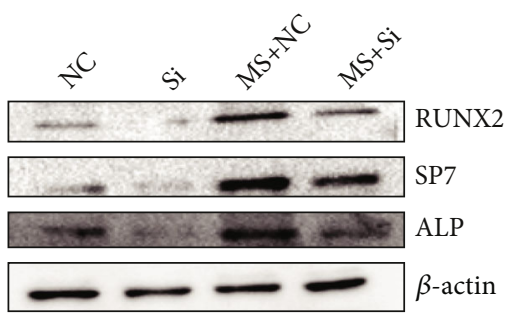

(d)

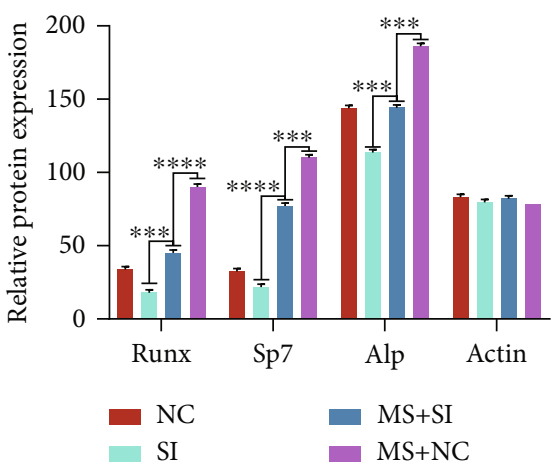

(e)

Figure 4: Effect of MAGT1 gene silencing on osteogenesis. (a) MAGT1 siRNA was transfected into BMSCs with an efficiency of 42\%, indicating successful transfection. (b) MAGT1 siRNA interference decreased ALP staining after 7 days, indicating that Mg ${ }^{2+}$-SMF induces osteogenesis via the MAGT1 gene. (c) MAGT1 siRNA interference decreased number of calcium nodules, suggesting that the MAGT1 gene plays a role in BMSCs osteogenesis and that $\mathrm{Mg}^{2+}$-SMF induces osteogenesis via the MAGT1 gene. (d) MAGT1 siRNA interference decreased the protein expression of Runx2, SP7, and ALP in BMSCs.

of calcium nodules between BMSCs treated with $\mathrm{Mg}^{2+}$ or the SMF (Figure 3(b)). Thus, the combination of $\mathrm{Mg}^{2+}$ and the SMF showed the best performance in promoting ECM mineralization.

To confirm that $\mathrm{Mg}^{2+}$ and the SMF increased osteogenesis, protein expression of various osteogenic markers was measured. As shown in Figures 3(c) and 3(d), the combination of $\mathrm{Mg}^{2+}$ and the SMF increased the expression levels of the COL1, RUNX2, Sp7, and ALP proteins to a greater extent than either treatment alone. Thus, $\mathrm{Mg}^{2+}$ and the
SMF synergistically increased the expression of representative osteogenic proteins in BMSCs.

To determine the effects of $\mathrm{Mg}^{2+}$ and the SMF on BMSC proliferation, cells were grown under different conditions, and their proliferation was measured on days $1,3,5$, and 7. Cell proliferation did not significantly differ among the groups on day 1 (Figure 3(e)). However, the proliferation of BMSCs treated with $\mathrm{Mg}^{2+}$ or the SMF and, particularly, with both $7.5 \mathrm{mM} \mathrm{Mg}^{2+}$ and $15 \mathrm{mT}$ SMF was higher than that in the control group on days 3 and 5 . Moreover, on 


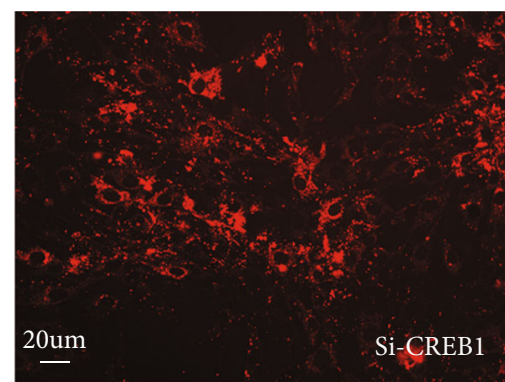

(a)
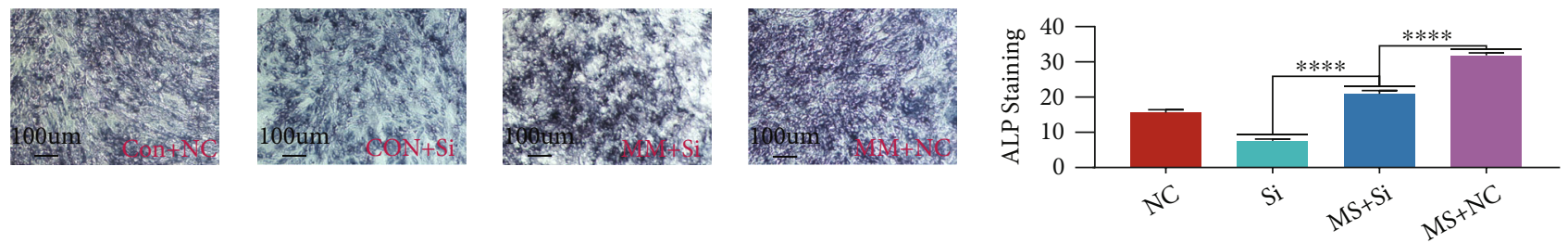

(b)
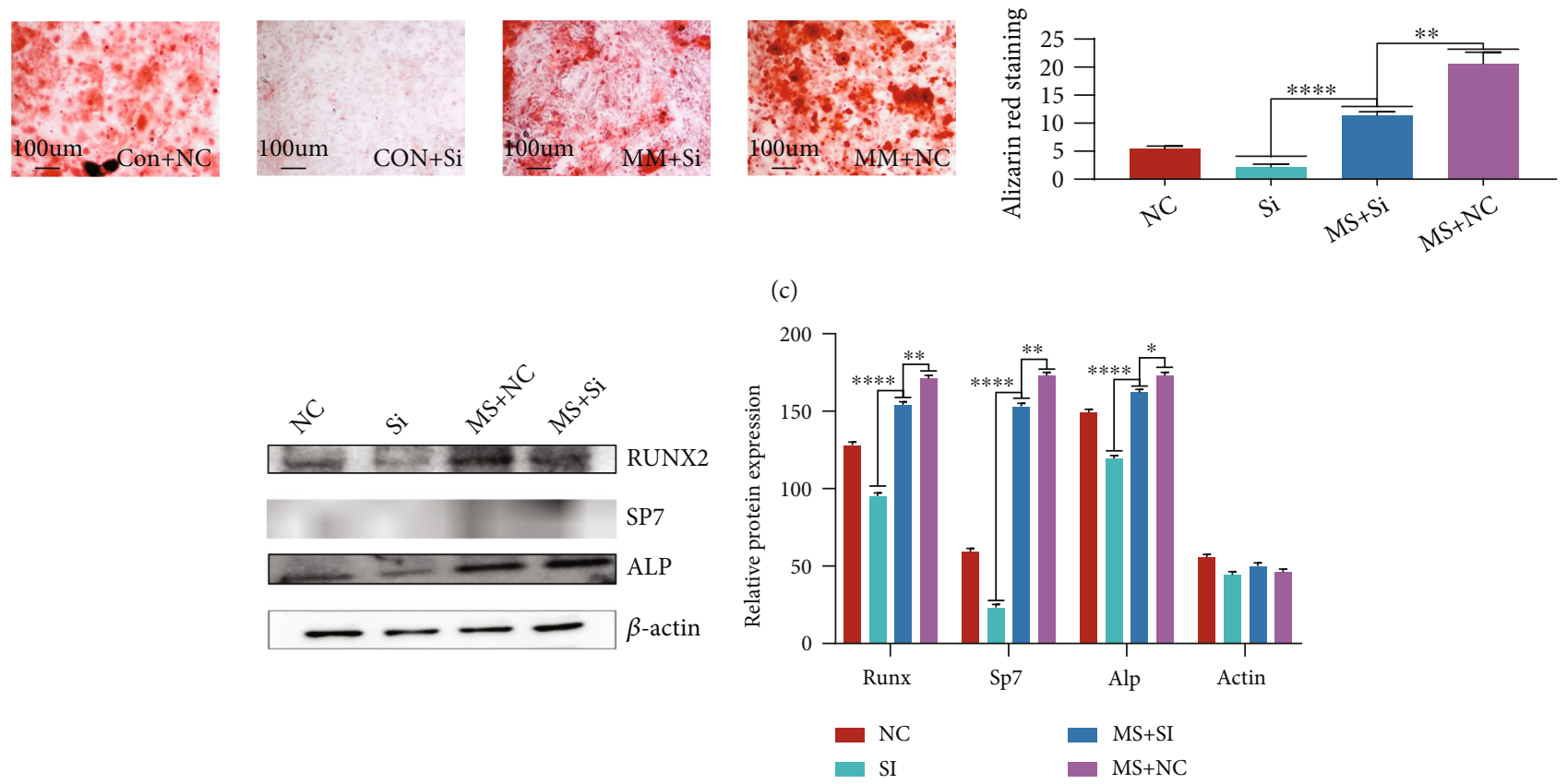

(d)

(e)

FIGURE 5: Effect of CREB1 gene silencing on osteogenesis. (a) CREB1 siRNA was transfected into BMSCs with an efficiency of 38\%, indicating successful transfection. (b) CREB1 siRNA interference decreased Alp staining, indicating that $\mathrm{Mg} 2+-\mathrm{SMF}$ induces osteogenesis via the CREB1 gene. (c) CREB1 siRNA interference decreased the number of calcium nodules, suggesting that the CREB1 gene plays a role in BMSCs osteogenesis. (d) CREB1 siRNA interference decreased the protein expression of Runx2, SP7, and ALP in BMSCs.

day 7, the proliferation was higher for BMSCs treated with both $\mathrm{Mg}^{2+}$ and the SMF than for those treated with $\mathrm{Mg}^{2+}$ and the SMF separately, and cell proliferation was higher in all three treatment groups than in the control group. Thus, the combination of $\mathrm{Mg}^{2+}$ and the SMF had the strongest proliferative effect on BMSCs. Taken together, these results showed that $\mathrm{Mg}^{2+}$ and the SMF synergistically enhanced the proliferation ability of BMSCs and their differentiation into osteoblasts compared with those in the groups treated with $\mathrm{Mg}^{2+}$ or SMF alone.
3.2. Effects of Magt1 and Creb1 Silencing on Osteogenesis. To investigate the roles of the MAGT1 channel and CREB1 protein in the synergistic osteogenic effects of $\mathrm{Mg}^{2+}$ and the SMF, both genes were silenced by transfecting BMSCs with the corresponding siRNAs. For Magt1 silencing, the siRNA transfection efficiency was $42 \%$ (Figure 4(a)). ALP staining (Figure 4(b)), Alizarin Red staining (Figure 4(c)), and western blot analysis (Figures $4(\mathrm{~d})-4(\mathrm{e})$ ) revealed that the levels of osteogenic markers were lower in the siRNA group than in the nonspecific control siRNA group and in the $\mathrm{Mg}^{2+}$ - 


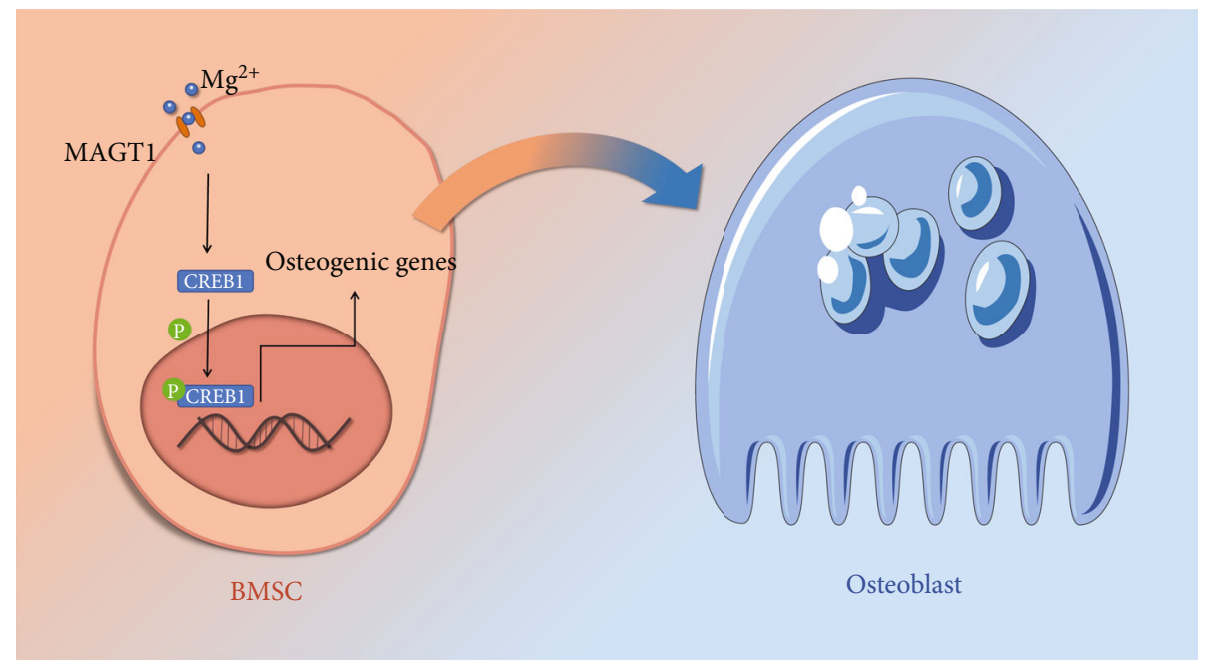

FIGURE 6: Osteogenic mechanism of $\mathrm{Mg}^{2+} \cdot \mathrm{Mg}^{2+}$ can activate CREB1 phosphorylation through the $\mathrm{Mg}^{2+}$ channel MAGT1 and promote osteogenic differentiation via transmembrane $\mathrm{Mg}^{2+}$ transport.

SMF-treated siRNA group than in the $\mathrm{Mg}^{2+}$-SMF-treated nonspecific control siRNA group. Taken together, these findings suggested that $\mathrm{Mg}^{2+}$ and the SMF induced osteogenesis in BMSCs via the MAGT1 channel. The siRNA transfection efficiency was 38\% (Figure 5(a)) in Crreb1 silencing. Similar findings were obtained in ALP staining (Figure 5(b)), Alizarin Red staining (Figure 5(c)), and western blot analysis (Figures 5(d)-5(e)) when Creb1 was silenced, indicating that the CREB1 protein also played a key role in the synergistic osteogenic mechanism of $\mathrm{Mg}^{2+}$ SMF. These data indicated that the MAGT1 channel and CREB1 protein played important roles in the synergistic osteogenic effects of $\mathrm{Mg}^{2+}$ and the SMF.

\section{Discussion}

$\mathrm{Mg}^{2+}$-based materials exert good osteogenic effects, and their mechanical properties are similar to those of the natural bone; however, these materials have not been applied clinically because of their high rate of degradation in vivo. $\mathrm{Mg}^{2+}$ can promote osteogenic differentiation by inducing CREB1 phosphorylation via the MAGT1 (Figure 6). According to this osteogenic property of $\mathrm{Mg}^{2+}$, we need to find a means to promote the influx to improve the utilization efficiency of $\mathrm{Mg}^{2+}$, so that only less $\mathrm{Mg}^{2+}$ is required to achieve a higher osteogenic effect. We found SMFs because it can change the opening frequency of $\mathrm{Mg}^{2+}$ channels to promote magnesium influx. Although SMFs can exert osteogenic effects via electrical and mechanical receptors on the cell membrane, it remains unclear whether the bioelectrical effects of a magnetic field could synergistically enhance osteogenic differentiation in combination with $\mathrm{Mg}^{2+}$. Herein, we combined $\mathrm{Mg}^{2+}$ and an SMF to explore whether they can synergistically enhance bone formation while eliminating the disadvantages of $\mathrm{Mg}^{2+}$-based biomaterials. Notably, we found that proliferation and osteogenic differentiation were significantly higher in BMSCs cotreated with $\mathrm{Mg}^{2+}$ and the SMF than in those treated with either intervention alone. Further gene silencing experiments suggested that these effects might be related to Magt1 and Creb1. Collectively, our data demonstrated that $\mathrm{Mg}^{2+}$ and the SMF synergistically promoted the proliferation and osteogenic differentiation of BMSCs via the MAGT1 channel and CREB1 protein.

Several related studies have clearly demonstrated that certain $\mathrm{Mg}^{2+}$ concentrations and certain SMF intensities can promote BMSC proliferation and osteogenesis. Similarly, we observed that a synergistic intervention with $\mathrm{Mg}^{2+}$-SMF significantly promoted the proliferation and induced osteogenic differentiation of BMSCs, which may be due to the effect of the SMF on MAGT1 channels on the cell membrane [38]. A previous study has shown that the opening frequency of ion channels on the cell membrane can change under the action of an SMF [39]. Therefore, SMFs can change the balance of the ion flow and membrane potential to promote bone formation and can also increase the opening frequency of MAGT1 channels to enhance the influx of $\mathrm{Mg}^{2+}$ to synergistically improve the osteogenic effect (Figure 7). Magnetic fields can modulate cellular functions, including cell morphology, cell cycle distribution, differentiation, proliferation, and gene expression [40]. This modulation may be due to electrodynamic interactions (Hall effect), magnetomechanical interactions, and radical pair effects [36]. Several studies have reported that different magnetic field environments have different effects on the cell differentiation ability. In particular, the effect of the magnetic field strength on cells has been a focus of research. As reported, S. Yamaguchi-Sekino, T. Kira, M. Sekino et al. found that the differentiation ability of cells was inhibited under the high-intensity magnetic field environment of $7 \mathrm{~T}$ [41]. They consider that SMFs may interfere with the opening of ion channels and hinder BMSC osteogenesis. However we found that a medium-strength $(15 \mathrm{mT})$ SMF promoted cell proliferation and osteogenesis. These differences are likely to be caused by two-way differences in the magnetic field strength in a cellular environment. At present, it is believed that the high-intensity magnetic field environment has an inhibitory effect on the differentiation ability of cells, whereas a medium-intensity magnetic field has a more 


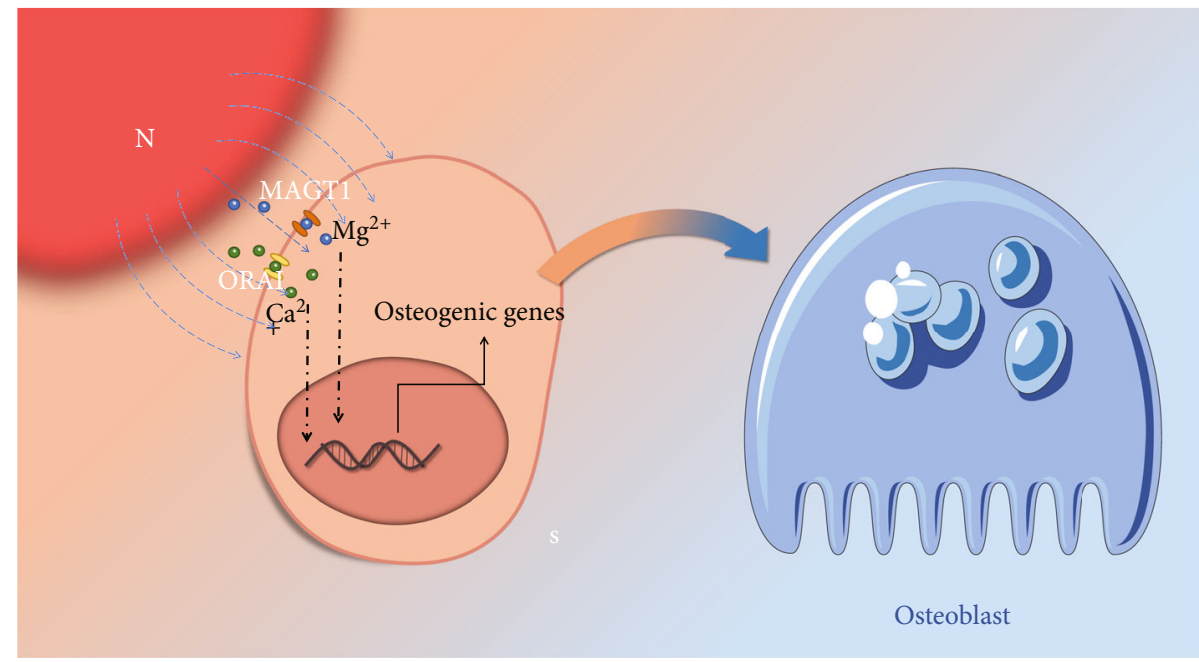

Figure 7: Mechanism of Mg2 +-SMF synergistic bone formation. Mg2 +-SMF increase the opening frequency of the MAGT1 channel, enhancing the influx of $\mathrm{Mg} 2+$ and thus the differentiation of BMSCs into osteoblasts.

beneficial biological effect. Our results confirmed that the medium-strength magnetic field was consistent with other articles to promote the osteogenic differentiation of BMSCs. In our experiments, compared with the control group, the SMF group showed stronger osteogenic properties in the results of ALP staining, Alizarin Red Staining and Western Blot.

Like the strength of the magnetic field, different types of magnetic fields have different effects on cell differentiation. A constant SMF with moderate intensity can induce the differentiation of BMSCs into osteoblasts by promoting the expression of related proteins. The sinusoidal electromagnetic field has a certain induction effect on the osteogenic differentiation of BMSCs, and the sinusoidal electromagnetic field of $1.0-2.0 \mathrm{mT}, 10-50 \mathrm{~Hz}$ has the greatest effect on the differentiation of bone marrow mesenchymal stem cells [42]. So we fixed the most suitable magnetic field strength environment for experiments.

Magnetic field position and orientation also have different effects on cell differentiation. As reported by Lin, S. Y, $\mathrm{Li}$, J et al., upon intervention of BMSCs in parallel and perpendicular magnetic field directions, the cells produced different differentiation states [43]. This may be due to the different angles of the magnetic poles and magnetic field lines to the cell. So we fixed the magnetic poles (using north magnetic poles for all interventions) to eliminate experimental errors.

To the best of our knowledge, this study is the first to demonstrate a synergistic effect between $\mathrm{Mg}^{2+}$ and the SMF in promoting BMSC proliferation and osteogenesis; however, the study has a few limitations. First, all experiments were performed using mouse BMSCs; therefore, further studies should examine the effects of $\mathrm{Mg}^{2+}$ and the SMF on BMSCs from other species, such as rabbits and humans. Similarly, since we only studied BMSCs cultured for 7 days, future experiments should extend the culture period and observe changes in osteogenic markers at different times. Finally, our research was based on the observations at the cellular level and thus may not reflect the process of bone formation in an organism. Subsequent studies are required to address these issues.

\section{Conclusions}

This study demonstrated that a medium-strength SMF intervention, combined with an appropriate $\mathrm{Mg}^{2+}$ concentration, could increase the expression of the COL1, Sp7, RUNX2, and ALP proteins in BMSCs via MAGT1 and CREB1 and significantly enhance the proliferation and osteoblast differentiation of BMSCs. Thus, $\mathrm{Mg}^{2+}$ and the SMF could be combined to develop new artificial bone materials with improved osteogenic properties to enhance bone cell proliferation and differentiation and promote the healing of bone defects. Future studies should identify the specific pathways through which $\mathrm{Mg}^{2+}$ and the SMF affect bone formation.

\section{Abbreviations}

MAGT1: Magnesium transporter 1

SMF: $\quad$ Static magnetic field

BMSCs: Bone mesenchymal stem cells

CCK-8 kit: Cell counting Kit-8

ALP: $\quad$ Alkaline phosphatase

CREB1: $\quad$ cAMP responsive element binding protein 1

PEMF: Pulsed electromagnetic fields

TREK1: $\quad$ TWIK-related $\mathrm{K}^{+}$channel 1

ORAI1/2: ORAI calcium release-activated calcium Modulator $1 / 2$.

\section{Data Availability}

The data used to support the findings of this study are included within the article.

\section{Conflicts of Interest}

The authors declare that they have no conflicts of interest. 


\section{Authors' Contributions}

Youwen Deng and Cijun Shuai designed this work. Yifan Wang and Xin Wu integrated and analyzed the data. Yifan Wang, Xin Wu and Pei Feng wrote this manuscript. Pei Feng and Wei Tan edited and revised the manuscript. All authors approved this manuscript.

\section{Funding}

This work was supported by The Natural Science Foundation of China (81472058, 52105352); Hunan Science and Technology Innovation Plan (2018SK2105, 422000008); Science and Technology Program of Huizhou (No.2020Y253); Medical Science and Technology Research Fund Project of Guangdong Province (B2021166); Postgraduate Research and Innovation Project of Central South University (1053320210754).

\section{Acknowledgments}

The authors thank the members in Hunan Engineering Laboratory for Orthopedic Biomaterials and the department of spine surgery of the Third Xiangya Hospital of the Central South University.

\section{Supplementary Materials}

Supplementary 1. rawdata-ALP: Alkaline phosphatase (ALP) staining by 5-bromo-4-chloro-3-indolyl-phosphate/NitroBlue-Tetrazolium ALP color development kit.

Supplementary 2. rawdata-cck8: Cell proliferation assay by cell counting kit (cck8).

Supplementary 3. rawdata-red: Alizarin red staining, osteoblast differentiation was measured by quantifying the formation of mineralized bone nodules using an alizarin red staining assay.

Supplementary 4. rawdata-siRNA: Silence genes in BMSCs by transfecting the cells with corresponding siRNAs.

Supplementary 5. rawdata-wb: Western blot analysis. ALP, Runx2, Osx, and Col-I protein expression were measured using western blotting.

\section{References}

[1] Z. P. Du, X. X. Feng, G. X. Cao et al., "The effect of carbon nanotubes on osteogenic functions of adipose-derived mesenchymal stem cells in vitro and bone formation in vivo compared with that of nano-hydroxyapatite and the possible mechanism," Bioactive Materials, vol. 6, no. 2, pp. 333-345, 2021.

[2] L. Wang, C. Y. Wang, S. Wu, Y. B. Fan, and X. M. Li, "Influence of the mechanical properties of biomaterials on degradability, cell behaviors and signaling pathways: current progress and challenges," Biomaterials Science, vol. 8, no. 10, pp. 2714-2733, 2020.

[3] M. Bessa-Goncalves, A. M. Silva, J. P. Bras et al., "Fibrinogen and magnesium combination biomaterials modulate macrophage phenotype, NF-kB signaling and crosstalk with mesen- chymal stem/stromal cells," Acta Biomaterialia, vol. 114, pp. 471-484, 2020.

[4] J. Nourisa, B. Zeller-Plumhoff, H. Helmholz, B. LuthringerFeyerabend, V. Ivannikov, and R. Willumeit-Romer, "Magnesium ions regulate mesenchymal stem cells population and osteogenic differentiation: a fuzzy agent-based modeling approach," Computational and Structural Biotechnology Journal, vol. 19, pp. 4110-4122, 2021.

[5] J. W. Park, T. Hanawa, and J. H. Chung, "The relative effects of ca and mg ions on MSC osteogenesis in the surface modification of microrough Ti implants," International Journal of Nanomedicine, vol. 14, pp. 5697-5711, 2019.

[6] Q. Wang, L. Xu, R. Willumeit-Romer, and B. J. C. LuthringerFeyerabend, "Macrophage-derived oncostatin M/bone morphogenetic protein 6 in response to mg-based materials influences pro-osteogenic activity of human umbilical cord perivascular cells," Acta Biomaterialia, vol. 133, pp. 268-279, 2021.

[7] Z. Zhai, X. Qu, H. Li et al., "The effect of metallic magnesium degradation products on osteoclast-induced osteolysis and attenuation of NF-kappaB and NFATc1 signaling," Biomaterials, vol. 35, no. 24, pp. 6299-6310, 2014.

[8] L. Zhang, C. Yang, J. Li, Y. Zhu, and X. Zhang, "High extracellular magnesium inhibits mineralized matrix deposition and modulates intracellular calcium signaling in human bone marrow-derived mesenchymal stem cells," Biochemical and Biophysical Research Communications, vol. 450, no. 4, pp. 1390-1395, 2014.

[9] Y. Zhang, J. Xu, Y. C. Ruan et al., "Implant-derived magnesium induces local neuronal production of CGRP to improve bone-fracture healing in rats," Nature Medicine, vol. 22, no. 10, pp. 1160-1169, 2016.

[10] W. Tang, Q. Liu, W. Tan, T. Sun, and Y. Deng, "LncRNA expression profile analysis of $\mathrm{mg}(2+)$-induced osteogenesis by RNA-seq and bioinformatics," Genes Genomics, vol. 43, no. 11, pp. 1247-1257, 2021.

[11] C. Y. Chang, W. Z. Lew, S. W. Feng et al., "Static magnetic field-enhanced osteogenic differentiation of human umbilical cord-derived mesenchymal stem cells via matrix vesicle secretion," International Journal of Radiation Biology, vol. 96, no. 9, pp. 1207-1217, 2020.

[12] G. Chen, Y. Zhuo, B. Tao et al., "Moderate SMFs attenuate bone loss in mice by promoting directional osteogenic differentiation of BMSCs," Stem Cell Research \& Therapy, vol. 11, no. 1 , p. $487,2020$.

[13] F. Javani Jouni, P. Abdolmaleki, and M. Movahedin, "Investigation on the effect of static magnetic field up to $15 \mathrm{mT}$ on the viability and proliferation rate of rat bone marrow stem cells," In Vitro Cellular \& Developmental Biology-Animal, vol. 49, no. 3, pp. 212-219, 2013.

[14] L. Kong, Y. Han, Q. Lu et al., "Polydopamine coating with static magnetic field promotes the osteogenic differentiation of human bone-derived mesenchymal stem cells on threedimensional printed porous titanium scaffolds by upregulation of the BMP-Smads signaling pathway," American Journal of Translational Research, vol. 12, no. 12, pp. 7812-7825, 2020.

[15] C. C. Lin, Y. T. Chang, R. W. Lin, C. W. Chang, G. J. Wang, and K. A. Lai, "Single pulsed electromagnetic field restores bone mass and microarchitecture in denervation/disuse osteopenic mice," Medical Engineering \& Physics, vol. 80, pp. 52-59, 2020. 
[16] F. Martini, A. Pellati, E. Mazzoni et al., "Bone Morphogenetic Protein-2 Signaling in the Osteogenic Differentiation of Human Bone Marrow Mesenchymal Stem Cells Induced by Pulsed Electromagnetic Fields," International Journal of Molecular Sciences, vol. 21, no. 6, 2020.

[17] Q. Ren, J. Zhou, M. G. Wang, and K. M. Chen, "Pulsed electromagnetic fields stimulating osteogenic differentiation and maturation involves primary cilia-PI3K/AKT pathway," Beijing Da Xue Xue Bao. Yi Xue Ban, vol. 51, no. 2, pp. 245-251, 2019.

[18] D. Wu, X. Chang, J. Tian et al., "Bone mesenchymal stem cells stimulation by magnetic nanoparticles and a static magnetic field: release of exosomal miR-1260a improves osteogenesis and angiogenesis," Journal of nanobiotechnology, vol. 19, no. 1, p. 209, 2021.

[19] Y. Naito, S. Yamada, Y. Jinno et al., "Bone-forming effect of a static magnetic field in rabbit femurs," The International Journal of Periodontics \& Restorative Dentistry, vol. 39, no. 2, pp. 259-264, 2019.

[20] Y. He, G. Chen, Y. Li et al., "Effect of magnetic graphene oxide on cellular behaviors and osteogenesis under a moderate static magnetic field," Nanomedicine, vol. 37, article 102435, 2021.

[21] M. Filippi, B. Dasen, J. Guerrero et al., "Magnetic nanocomposite hydrogels and static magnetic field stimulate the osteoblastic and vasculogenic profile of adipose-derived cells," Biomaterials, vol. 223, article 119468, 2019.

[22] S. K. Boda, G. Thrivikraman, and B. Basu, "Magnetic field assisted stem cell differentiation - role of substrate magnetization in osteogenesis," Journal of Materials Chemistry B, vol. 3, no. 16, pp. 3150-3168, 2015.

[23] C. Androjna, C. S. Yee, C. R. White et al., "A comparison of alendronate to varying magnitude PEMF in mitigating bone loss and altering bone remodeling in skeletally mature osteoporotic rats," Bone, vol. 143, article 115761, 2021.

[24] J. Cai, W. Li, T. Sun, X. Li, E. Luo, and D. Jing, "Pulsed electromagnetic fields preserve bone architecture and mechanical properties and stimulate porous implant osseointegration by promoting bone anabolism in type 1 diabetic rabbits," Osteoporosis International, vol. 29, no. 5, pp. 1177-1191, 2018.

[25] M. M. Eid, A. M. El-Gendy, W. K. Abdelbasset, S. M. Elkholi, and M. S. Abdel-Fattah, "The effect of magnetic therapy and moderate aerobic exercise on osteoporotic patients: a randomized clinical study," Medicine (Baltimore), vol. 100, no. 39, article e27379, 2021.

[26] G. Qian, M. Wang, Y. Dong, Y. Hong, Y. Yu, and J. Mei, "Effect of combined treatment with pulsed electromagnetic field stimulation and sclerostin monoclonal antibody on changes in bone metabolism and pedicle screw augmentation in rabbits with ovariectomy-induced osteoporosis," Annals of Palliative Medicine, vol. 10, no. 2, pp. 1070-1078, 2021.

[27] L. Wang, Y. Li, S. Xie, J. Huang, K. Song, and C. He, "Effects of pulsed electromagnetic field therapy at different frequencies on bone mass and microarchitecture in osteoporotic mice," Bioelectromagnetics, vol. 42, no. 6, pp. 441-454, 2021.

[28] W. Li, S. Zhao, W. He, M. Zhang, S. Li, and Y. Xu, "Static magnetic fields accelerate osteogenesis by regulating FLRT/BMP pathway," Biochemical and Biophysical Research Communications, vol. 527, no. 1, pp. 83-89, 2020.

[29] Z. Bao, M. Fan, L. Ma, Q. Duan, and W. Jiang, "The effects of pulsed electromagnetic fields combined with a static magnetic intramedullary implant on the repair of bone defects: a preliminary study," Electromagnetic Biology and Medicine, vol. 38, no. 3, pp. 210-217, 2019.
[30] K. Marycz, K. Kornicka, and M. Rocken, "Static magnetic field (SMF) as a regulator of stem cell fate-new perspectives in regenerative medicine arising from an underestimated tool," Stem Cell Reviews and Reports, vol. 14, no. 6, pp. 785-792, 2018.

[31] Z. Huang, Y. He, X. Chang et al., "A magnetic iron oxide/Polydopamine coating can improve osteogenesis of 3D-printed porous titanium scaffolds with a static magnetic field by upregulating the TGFbeta-Smads pathway," Advanced Healthcare Materials, vol. 9, no. 14, article e2000318, 2020.

[32] X. Li, Q. Zou, Y. Man, and W. Li, "Synergistic effects of novel superparamagnetic/Upconversion $\mathrm{HA}$ material and $\mathrm{Ti} / \mathrm{mag}$ net implant on biological performance and long-term in vivo tracking," Small, vol. 15, no. 31, article e1901617, 2019.

[33] L. Hao, L. Li, P. Wang et al., "Synergistic osteogenesis promoted by magnetically actuated nano-mechanical stimuli," Nanoscale, vol. 11, no. 48, pp. 23423-23437, 2019.

[34] H. Zhang, L. Gan, X. Zhu et al., "Moderate-intensity 4mT static magnetic fields prevent bone architectural deterioration and strength reduction by stimulating bone formation in streptozotocin-treated diabetic rats," Bone, vol. 107, pp. 3644, 2018.

[35] A. Russo, M. Bianchi, M. Sartori et al., "Magnetic forces and magnetized biomaterials provide dynamic flux information during bone regeneration," Journal of Materials Science. Materials in Medicine, vol. 27, no. 3, p. 51, 2016.

[36] J. Zhang, C. Ding, L. Ren, Y. Zhou, and P. Shang, "The effects of static magnetic fields on bone," Progress in Biophysics and Molecular Biology, vol. 114, no. 3, pp. 146-152, 2014.

[37] J. M. Kanczler, H. S. Sura, J. Magnay et al., "Controlled differentiation of human bone marrow stromal cells using magnetic nanoparticle technology," Tissue Engineering. Part A, vol. 16, no. 10, pp. 3241-3250, 2010.

[38] J. Zheng, X. Mao, J. Ling, C. Chen, and W. Zhang, "Role of magnesium transporter subtype 1 (MagT1) in the osteogenic differentiation of rat bone marrow stem cells," Biological Trace Element Research, vol. 171, no. 1, pp. 131-137, 2016.

[39] D. Mohanta, E. Stava, M. Yu, and R. H. Blick, "Creation and regulation of ion channels across reconstituted phospholipid bilayers generated by streptavidin-linked magnetite nanoparticles," Physical Review. E, Statistical, Nonlinear, and Soft Matter Physics, vol. 89, no. 1, article 012707, 2014.

[40] J. Miyakoshi, "Effects of static magnetic fields at the cellular level," Progress in Biophysics and Molecular Biology, vol. 87, no. (2-3), pp. 213-223, 2005.

[41] S. Yamaguchi-Sekino, T. Kira, M. Sekino, and M. Akahane, "Effects of $7 \mathrm{~T}$ static magnetic fields on the expression of biological markers and the formation of bone in rats," Bioelectromagnetics, vol. 40, no. 1, pp. 16-26, 2019.

[42] F. Luo, T. Y. Hou, Z. H. Zhang, Z. Xie, X. H. Wu, and J. Z. Xu, "Effects of pulsed electromagnetic field frequencies on the osteogenic differentiation of human mesenchymal stem cells," Orthopedics, vol. 35, no. 4, pp. E526-E531, 2012.

[43] S. Y. Lin, J. Li, J. Q. Shao et al., "Anisotropic magnetomechanical stimulation on collagen coatings to accelerate osteogenesis," Colloids and Surfaces B-Biointerfaces, vol. 210, p. $112227,2022$. 